It is evident from these results that the calculated constants vary usually irregularly and but little.

As this is the case with two substances of so widely different chemical nature and physical properties as benzoic acid and lead chloride, it is safe to assume that the law is a general one. It may be expressed as follows: The rate at which a solid substance dissolves in its own solution is proportional to the difference between the concentration of that solution and the concentration of the saturated solution.

Massachusetts INstitete of Technology, Boston, MAY, I897.

\title{
THE ANALYSIS OF BEARING-METAL ALLOYS, WITH A NEW VOLUMETRIC METHOD FOR DETERMINING COPPER. ${ }^{1}$
}

\author{
BY W, E. Garrigues.
}

Received October 28,1897 .

$\int U$ UDGING by the number of articles that continue to appear from year to year in chemical journals, elaborating more or less complete schemes for the analysis of these alloys, the subject is still a timely one for discussion. There is, furthermore, small likelihood that this apparent interest will $\mathrm{lag}$, in the near future at least, owing to the evident fact-and it is one that must not be lost sight of in passing judgment on the merit of such a combination of methods for separation and determination as the present paper deals with-that we are not considering a material capable of being handled like steel, where accurate methods have been found for ascertaining the quantity of almost every element present without the necessity of first removing three or four others from solution.

In the light of our present knowledge of the chemical deportment of the metals of the hydrogen sulphide and ammonium sulphide groups, all methods which even approach accuracy must of necessity be comparatively tedious. Not alone is it essential in almost every case to free a solution from interfering elements to reach the one sought, but in very many instances it is all but impossible to get the former out of the way without sustaining loss of the latter. Add to this the manipulative difficulties such as the washing of slimy precipitates that persist in I Read before the Chemical Section of the Engineers Society of Western Pennsylvania. 
passing the filter medium, and those that in coming down occlude non-volatile salts used as reagents or sustain loss in ignition through reduction and volatilization-there is certainly sufficient difficulty remaining to interest many of us for years to come.

The present paper is not written so much with the object of presenting innovations that conduce to speed as to discuss some of the inaccuracies in very commonly used processes and to considering means for overcoming them, even though at times additional labor may be involved. Of late years the art of successfully working scrap has reached such perfection that it is probably not an error to assume that the greater part of the metal in alloy bearings now in use, was at one time doing duty along a varied line reaching from wash-boiler bottoms to tea packages. The inevitable result is that the subject has grown still more intricate from the analyst's point of view, the range of elements existing in small percentages being greater.

The alloys considered are bronze, brass, and white metal, composed of copper, tin, antimony, lead, zinc, iron, phosphorus, and arsenic.

The advantage of a qualitative analysis previous to beginning the analysis proper, has often been dwelt upon, and there is but little doubt that it almost invariably repays the trouble tenfold. In the scheme to be outlined here it is particularly desired to know beforehand whether antimony, iron, zinc, and arsenic are absent; for this purpose we have found the following procedure easy and satisfactory. The sample is oxidized with nitric acid and evaporated dry, boiled with a little dilute nitric acid, the residue filtered out and washed.

Antimony.-Ignite a portion of the residue intensely, boil with strong hydrochloric acid, and dilute with an equal volume of water. Filter and add considerable water, when a white precipitate indicates antimony. Confirm by orange hydrogen sulphide precipitate.

Arsenic and Iron.-The remaining portion of the residue is warmed with a little caustic soda solution and dissolved by the further addition of alkali sulphide and heating. The black residue is removed by filtration and treated for iron by any suitable 
method. In the alkaline filtrate arsenic is precipitated with ammonia and magnesia mixture, the precipitate filtered out and dissolved in hydrochloric acid, which solution is boiled with sulphurous acid, and then saturated with hydrogen sulphide, when the arsenic, if present, is obtained as sulphide.

Zinc.-The filtrate from the nitric acid insoluble residue is saturated with bydrogen sulphide, the filtrate mixed with onetenth its volume of strong hydrochloric acid, and potassium ferrocyanide added, when zinc is indicated by a white precipitate of the ferrocyanide.

Quite a large amount of the sample should be used, as otherwise antimony may all pass into the nitric acid filtrate and thus escape detection. The ignited stannic oxide is entirely insoluble in the boiling hydrochloric acid, while sufficient antimony dissolves to give ample reactions. The large amount of hydrochloric acid is added previous to testing for zinc, to prevent the coprecipitation of any lead that may have escaped coming down as a result of the hydrogen sulphide treatment.

Coming now to the quantitative part of our subject, we have to consider first

\section{THE DETERMINATION OF SINGLE ELEMENTS.}

Zinc, when present in fairly large quantity, and an absolutely correct determination is not required, can be very conveniently measured by titration with standard potassium ferrocyanide, but in smaller anounts a gravimetric method is preferable. For the details of the process, see Stone.' The end point is marked by an immediate green color with cobalt nitrate.

Stone directs that the volume of standard liquor necessary to give the end reaction be determined by a blank experiment, and that this amount be subtracted from each titration. This statement we have been entirely unable to verify, as witness the following set of titrations of varying amounts of the same solution of zinc chloride:

1 This Journal, 17, 413. 


\begin{tabular}{|c|}
\hline $\begin{array}{l}\text { Zinc chlo- } \\
\text { ride. } \\
\text { cc. }\end{array}$ \\
\hline 5 \\
\hline Io \\
\hline 12.5 \\
\hline 15 \\
\hline 25 \\
\hline
\end{tabular}

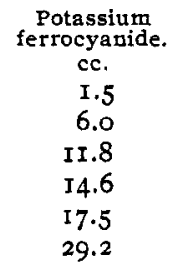

Tota1
Per cent.
$\cdots .$.
30.0
29.5
29.2
29.15
29.2

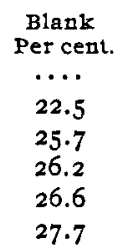

Total per cent. column is figured on the assumption that the highest titration is per cent. zinc, the lower titrations then being multiplied to equal the same volume of zinc chloride used-no blank being subtracted. Figures in blank per cent. column are obtained in the same manner except that the blank is in each case subtracted. It will be noticed that the figures in the latter column are anything but uniform among themselves. To get the best results the standard solution should be titrated against a known zinc solution of the same strength as the sample under examination. In applying the process absence of copper and iron is essential.

When the conditions of precipitation are fully understood one of the neatest and most accurate analytical processes is the determination of zinc as phosphate. All metals, inclusive of alkalies, should be absent.

The solution should be exactly neutralized with hydrochloric acid or ammonia, as the case may be, using methyl orange as indicator, unless the liquid contains acetic acid, in which case no further inconvenience results except that litmus is substituted for methyl orange. A very large excess of ammonium phosphate is to be avoided, as otherwise the precipitate is redissolved to some extent. This part of the process is, however, not the delicate matter it might seem from the statement, as will be shown later. The most satisfactory mode of procedure is to add first say one gram of the phosphate, which will not hold up the smallest traces of zinc, and if a large precipitate comes down, to continue the addition. Hydrogen sulphide should give no trace of precipitate in the filtrate. It is highly advisable to work only with ammonium salts from the beginning, sodium salts being less good, and potassium salts entirely prohibited as they are carried down with the precipitate by occlusion in astonishing amounts. 
The ammonium phosphate is added to the warm solution of the zinc, which is then further warmed until the precipitate is entirely free from any flocks and the solution has completely cleared. Filtering on a Gooch crucible, washing with water and then alcohol, drying at $100^{\circ} \mathrm{C}$, and weighing as zinc ammonium phosphate is recommended. Using an ordinary filter paper and igniting seems to cause an unavoidable loss through reduction, no matter what care be exercised in separating the filter. It may, however, be ignited with perfect safety on a Gooch, even though a disk of paper be used as the filtering medium. In the latter case the paper should be turned above the precipitate toward the mouth of the crucible.

The details of the method, with sodium phosphate as precipitant, appear in the last edition of "Crookes' Select Methods." The original author's statement that the ignition gives low results owing to volatility of the zinc pyrophosphate cannot be admitted, in fact our excellent results obtained by ignition on a Gooch prove that such is not the case. The same applies to his claim that the precipitation takes place in an acid solution.

Though a difference of opinion as to what constitutes an acid solution may in some cases exist, it certainly does not seem reasonable to apply the term to a liquid smelling strongly of ammonia. The reactions occurring are probably expressed by the equations

a. $\mathrm{ZnCl}_{2}+\left(\mathrm{NH}_{4}\right)_{2} \mathrm{HPO}_{4}=\mathrm{ZnHPO}_{4}+2 \mathrm{NH}_{4} \mathrm{Cl}$.

b. $\mathrm{ZnHPO}_{4}+\left(\mathrm{NH}_{4}\right)_{2} \mathrm{HPO}_{4}=\mathrm{ZnNH}_{4} \mathrm{PO}_{4}+2 \mathrm{NH}_{4} \mathrm{H}_{2} \mathrm{PO}_{4}$.

It is even likely that these two stages are entirely separate, since in a cold solution the precipitate is at first as flocculent as an alumina precipitate, while on warming it becomes as dense as barium sulphate. If only the amount of ammonium phosphate called for by the first equation is added, the precipitate remains flocculent despite continued heating.

The double equation requires nearly five parts ammonium phosphate for one part zinc and we have successfully thrown down two-tenths gram zinc with one gram of the phosphate obtaining it all as the ammonium salt. The same quantity was also perfectly precipitated with five grams of ammonium phos. 
phate but eight grams left a little zinc in solution. Similarly, 0.008 gram was completely thrown down with 0.05 and 3.00 grams, respectively, while five grams caused some re-solution.

Returning to the question of acidity, it will be seen from the equation that monoammonium phosphate remains as a product of the reaction but the excess of the diammonium phosphate, always used in practice, decomposes on heating to monoammonium phosphate and free ammonia, thus determining the alkalinity of the liquid. The precipitate is soluble with great facility in any kind of acid or in excess of ammonia. Too much cannot be said in favor of the process for accurate work.

Copper is determined in smaller quantities by the iodide method and in larger quantities by the new process described below. The latest literature added to the iodide method is Low's paper. ${ }^{1}$ He introduces two modifications : Oxidation of any arsenic present with potassium chlorate and neutralization of the excess of nitric acid with zinc acetate.

There is no doubt, as Low states, that the end point is better as a result of the use of zinc acetate than when the sodium salt is employed; the oxidation with chlorate is also a great step in advance for the process. It is, however, a mistake to use any acetate as the end point is then at best not clear, though with practice it can be distinguished.

Some time ago the writer had the honor to present to this section a paper on the iodide method for copper, in which it was proposed to avoid the inaccuracy introduced by the presence of arsenic by separating the copper from it with glucose in the wellknown manner. The object was to retain the clear end point when the titration is conducted in a very slightly acid sulphuric acid solution. Since that time the following, relative to the subject, has been ascertained :

When copper and arsenic, either as metals or sulphides, as obtained in the course of analysis, are dissolved in nitric acid, there is very apt to result a solution of the arsenic both as arsenic and arsenious acid. If now this solution be boiled with chlorate in a fairly concentrated condition, all the arsenic is oxidized to the higher form. Arsenic acid in strongly mineral acid solution, mixed with an iodide, is reduced to arsenious acid 1 This Journa1, 18,458 . 
with liberation of iodine, causing the result for copper to appear high. In a solution which is, however, only faintly acid from a mineral acid, this reaction does not take place, in fact free iodine will then even oxidize arsenious acid to some extent but far less so than in acetic solution. In the presence of little acid and arsenious acid the result for copper would therefore appear low, since the iodine liberated by the copper would be in part consumed by the arsenic instead of being measured by the titrating liquid. If the copper solution be only faintly acid and the arsenic all present as arsenic acid, an accurate result for copper is obtained in sulphuric acid solution and the end point with starch is vastly superior than if either zinc or sodium acetate has been used.

The thiosulphate solution should be standardized on approximately the amount of copper found in the assay, conveniently obtained by measuring out a standard copper sulphate solution, if the best results are to be gotten. In the writer's experience a little more than the theoretical amount of iodine is liberated, and this not in absolutely uniform ratio. For small amounts of copper it is advisable to allow the solution to stand a given time, say ten minutes, between the addition of the iodide and the titration, otherwise the end point will come and go in a rather unsatisfactory manner.

While the method is good, very much to be preferred to the cyanide titration of Parks and Mohr, after an extended experience with it we must certainly dissent from Low's claim of superiority for it as against the electrolytic assay.

NEW PROCESS FOR COPPER.

This depends on the precipitation of the copper as cuprous thiocyanate according to Rivot, and the alkalimetric determination of the combined thiocyanic acid.

The solution is evaporated with as little sulphuric acid as possible to expel any volatile acids; warm gently after dilution, and add sulphurous acid until its presence is plainly apparent by odor. Precipitate with an alkali thiocyanate. When the liquid settles perfectly clear, which is promoted by further warming, filter and wash well with water.

Transfer the filter and contents back to the beaker in which the precipitation was effected, boil with a measured excess of 
standard caustic alkali, a few minutes is sufficient, then cool and dilute to a convenient bulk $(200 \mathrm{cc}$.). After passing through a dry filter, take of the filtrate a quantity equal to one-half the bulk of the original solution, and titrate to neutrality with standard acid and methyl orange.

The rationale of the process is that caustic alkali converts cuprous thiocyanate into cuprous hydroxide and alkali thiocyanate, the latter body being neutral to methyl orange. The reaction proceeds quantitatively according to the equation

$$
{ }_{2} \mathrm{CuSCN}+2 \mathrm{NaOH}=\mathrm{Cu}_{2}(\mathrm{OH})_{2}+2 \mathrm{NaSCN} \text {, }
$$

with all quantities of copper, one equivalent of caustic soda equaling one of copper. No requirement of an empirical standard is one of the advantages of the process. The writer claims for it greater accuracy than the iodide method is capable of.

Antimony is determined by Mohr's method: titration with iodine in alkaline solution, antimonic acid being previously reduced by the method of Gooch and Gruener.' This is very much to be preferred to the oxidimetric method again recently advocated by Thompson, ${ }^{2}$ or to any attempt at separation from tin.

Lead is determined as sulphate, filtering on a Gooch with a paper disk, drying and weighing. In this manner the determination is capable of great accuracy, the drying being much better than ignition.

Tin is weighed as oxide, or in presence of antimony is determined by difference in weight.

Arsenic by Lundin's distillation process as described by Blair in his work on iron analysis.

Phosphorus as magnesium pyrophosphate.

GENERAL, CONSIDERATIONS.

When an alloy is treated with nitric acid, a more or less white residue remains insoluble. This contains all the tin, part of the antimony, iron, arsenic, phosphorus, copper, and lead, the two last of course only in small amount though the copper may amount to several milligrams. No method of treatment has

$1 \mathrm{Am} . J . S c i, 42,233$.

2 J. Soc. Chem. Ind., I5, 255. 
been found which gives this residue free from iron and copper. If all excess of nitric acid be evaporated and the residue again taken up with dilute nitric acid it is possible to retain all the iron in the residue, provided the tin is present in fair amount. The same applies also to the arsenic and antimony, though it is probably unsafe to count on this. In no event can the insoluble residue from a brass or bronze be considered sufficiently pure to weigh as stannic oxide. We separate the iron, copper, and lead from the tin and antimony by fusing with sodium thiosulphate and dissolving in water, preferring this to attempting to make the usual alkali sulphide separation in solution with all the copper and lead. The thiosulphate is a neater and quicker flux than sodium carbonate and sulphur.

If to save time in determining tin alone, when phosphorus, arsenic, and antimony are absent, it is desired to obtain the weight of stannic oxide by difference, the nitric acid insoluble residue may be ignited and weighed, fused with thiosulphate, dissolved in water, and the sulphides of lead, copper, and iron remaining, removed by filtratiun. The filter is then moistened with nitric acid and ignited in the same crucible (the fusion does not alter its weight perceptibly), subtracting the weight so found from the total weight of the residue. The difference is taken as stannic oxide.

A slight error must, however, be noted here: any lead present was weighed first as oxide and afterwards subtracted partly or wholly as sulphate. It may be present to an extent that will make this error serious. If, on the other hand, the sulphides are not moistened with nitric acid, the iron is very likely to burn only to the magnetic oxide, where it was at first weighed as the peroxide, while the condition of the lead is indefinite.

In the case of a complete analysis of an alloy containing zinc it is a great help to get all the iron out of the way at this early stage, and as the copper and lead must be returned to the main solution it will be more troublesome to separate these after igniting the sulphide precipitate than to precipitate the tin directly as sulphide from the solution of the fusion, and converting to oxide for weighing. The latter also has the advantage that it is applicable in the presence of phosphorus. 
Hempel, ${ }^{1}$ referring to the separation of phosphorus from tin, states that some of the phosphorus invariably comes down with the hydrogen sulphide precipitate of the tin. Whether or not the same applies to the sulphide precipitate obtained as a result of acidulating the dissolved fusion we have not ascertained, but it seems probable that it would not. At any rate the amount is too small to practically affect a tin determination in phosphor bronze. Arsenic, however, will largely remain with the ignited tin precipitate unless previously separated.

When the nitric acid filtrate from the insoluble residue is evaporated with a little sulphuric acid to take out the lead, there results a further precipitation of antimony. The lead sulphate cannot be removed by solution in ammonium acetate, as the antimonic acid is likewise affected. A large excess of sulphuric acid prevents this second precipitation, but its presence is undesirable for the separation of copper as thiocyanate. A little tartaric acid added to the diluting water will also prevent it, and has, moreover, no effect on the copper precipitate, but this may cause the re-solution of a trace of lead. For ordinary purposes this amount is negligible, but at any rate it may be very easily recovered and added at a later stage.

Alloys containing antimony are usually poor in copper and free from zinc; exceptions, however must be allowed for, and in the presence of both zinc and antimony the precipitation of copper as thiocyanate is not advisable, as the scheme thereby becomes complicated. Instead, the excess of sulphuric acid is used to prevent the precipitation, with the lead sulphate, of any antimony that remains in the nitric acid solution. In the filtrate from the lead sulphate the antimony and copper are thrown down together as sulphides and separated with caustic soda. In the residue the copper may, of course, be determined by the thiocyanate process, but as it is free from any other metals it is directly available for titration by the iodide method. In the filtrate from the sulphides the zinc is obtained as phosphate. It should be remembered that if great accuracy is desired in the figure for zinc, the sulphide precipitate, after removing the antimony, is to be dissolved and again precipitated to insure against loss of zinc.

1 Ber. d. chem. Ges., 22, 2478. 
As a result of converting the mixed sulphides of tin and antimony into oxides for weighing, a slight error is sustained owing to the fact that the two sulphides require different treatment to give accurate results. By treatment with fuming nitric acid combined sulphur is oxidized completely and the full heat of a good Bunsen burner will convert all pentoxide of antimony to tetroxide without any trouble, but while the tin precipitate requires a white heat to drive out the last traces of water, antimony will not stand this heat. If, therefore, the Bunsen flame be used and one-tenth per cent. be subtracted from each twenty per cent. of tin found, the result is better than if the actual weight obtained is taken.

As the sulphides of tin and antimony are recovered from the dissolved fusion, they are mixed with considerable free sulphur which cannot be completely oxidized with fuming nitric acid in one evaporation. For the most careful work, washing the dried sulphides with carbon disulphide is necessary, but ordinarily, careful ignition will answer. After evaporating the mass to dryness with fuming nitric acid and burning at a temperature just sufficient to ignite the sulphur, the residue is again moistened with fuming nitric acid for final ignition. This is important. The paper is, of course, to be consumed separately. A well-rounded, inverted lid is used as a cover during the action of the acid.

When the ignited oxides of tin and antimony are dissolved by fusion with caustic (or carbonated) alkali, and obtained as higher chlorides in acid solution, potassium iodide reduces only the antimonic salt. We prefer to boil down twice to about fifty cc. to insure the expulsion of the liberated iodine. We have found the color of the liquid not a safe guide in practice except that if perceptible deepening of the yellow takes place when approaching the bulk mentioned, the liquid must be immediately diluted and the second evaporation not carried quite so far. Failure to do this will result in loss of antimony by volatilization.

The separation with hydrogen sulphide of copper from zinc in hydrochloric acid is, according to Fresenius, rarely perfect. On testing a number of copper sulphide precipitates from solutions of brass containing fifteen cc. strong hydrochloric acid in a bulk of $200 \mathrm{cc}$. of liquid, we have never failed to find zinc in appreciable quantity. The precipitation as thiocyanate is, on the other 
hand, absolutely free from this source of error ; and if the volatile acids are expelled with as small a quantity of sulphuric acid as possible, the amount of copper that escapes precipitation is infinitesimal. The precipitate cannot be dried and weighed with anywhere near the accuracy one might be led to suppose from the single experiment quoted by Fresenius. Such a procedure is quite out of the question.

The excess of ammonium thiocyanate exerts no deleterious action on the zinc precipitation with ammonium phosphate in the filtrate, and methyl orange is available as indicator for exactly neutralizing the solution. When potassium thiocyanate is used the zinc precipitate is highly contaminated, so much so that it fuses at a low heat to a clear liquid, the results being entirely without value.

\section{THE ANAIYSIS OF BRONZE AND BRASS. (ANTIMONY BEING}

\section{ABSENT.)}

Oxidize one gram with strong nitric acid and evaporate on the water-bath to complete expulsion of the acid. Take up with fifty cc. three per cent. nitric acid and filter after boiling five minutes. Ignite filter and contents together in a porcelain crucible, grind the residue with a thick glass rod, and fuse with a liberal addition of sodium thiosulphate. Leach out with a little water, digest until the liquid is clear yellow, and remove the precipitated sulphides by filtration. In the absence of arsenic, determine tin in the filtrate by acidulating with hydrochloric acid, filtering out the sulphide of tin, and washing with ammonium acetate. Ignite at low temperature, together with the filter, raising the heat gradually after all free sulphur has been consumed, and finishing with a blast to constant weight.

The mixed sulphides of lead, copper, and iron, recovered from the fusion, are dissolved by boiling with twenty per cent. nitric acid, from which solution the iron is thrown down by ammonium acetate. The filtrate is added to the main solution and the whole evaporated with as little sulphuric acid as possible, the lead sulphate collected on a Gooch crucible, and weighed.

In the filtrate the copper is thrown down as thiocyanate and its value determined by the method described. The zinc is then 
obtained from the neutralized filtrate with ammonium phosphate, observing the precautions mentioned.

Phosphorus is determined in a separate portion by heating some time with aqua regia, passing hydrogen sulphide into the alkaline solution, and filtering. The phosphorus is then obtained with magnesia mixture as usual. If qualitative tests have shown presence of arsenic the magnesia precipitate is dissolved in hydrochloric acid, arsenic reduced by boiling with sulphurous acid, and precipitated with hydrogen sulphide. In the filtrate the phosphorus is obtained with molybdate and the yellow precipitate weighed, after collecting on a Gooch crucible with a paper disk.

Arsenic is determined in a separate portion by Lundin's method, just as described by Blair, except that we deem one evaporation to dryness with sulphuric acid sufficient.

In the tin determination, arsenic is separated from the solution of the thiosulphate fusion with magnesia mixture, the tin being then obtained in the filtrate as described.

In the analysis of brass, a more rapid, but not so accurate, determination of the copper and zinc may be obtained by using the iodide method for the former and the ferrocyanide method for the latter. The foregoing process is carried out to the point of obtaining the filtrate from the lead sulphate. This is mixed with about ten cc. hydrochloric acid and the copper precipitated by boiling a few minutes with strips of sheet aluminum. For a neat manner of treating this copper, consult Low's paper already referred to.

The filtrate is ready for titration of zinc with ferrocyanide according to Stone.

THE ANALYSIS OF WHITE METAL. (ANTIMONY BEING PRESENT.)

Proceed as in analysis of bronze up to the point of evaporation with sulphuric acid to remove lead, but in this case use about fifteen cc. of acid instead of about two. Dilute with eighty-five cc. water and filter without unnecessary delay or boiling. Dry and weigh the lead sulphate.

Copper and antimony are thrown down in the solution with hydrogen sulphide, and after boiling the filtrate free from the 
gas and oxidizing any fine sulphur with nitric acid, zinc is obtained as phosphate in the neutral solution.

The sulphides of copper and antimony are washed from the filter back into the beaker, boiled with a little caustic soda which is passed through the filter, and the filtrate now containing the antimony is added to the solution of the thiosulphate fusion of the nitric acid insoluble residue. The remaining copper sulphide is dissolved by boiling it in $1.20 \mathrm{sp}$. gr. nitric acid, when it is neutralized and the copper determined with potassium iodide and thiostulphate.

The alkaline solution, containing now all the tin and antimony, is acidulated with hydrochloric acid and the mixed sulphides filtered out. Ammonium acetate is used for washing as before, but the paper is separated from the dried precipitate and burned alone, followed by the precipitate itself. Ignite in the manner already described for antimony and tin sulphides in the presence of free sulphur. Having obtained the weight of the mixed oxides, it now remains only to determine the antimony, which is attained quite accurately, as follows:

Grind the residue in the crucible and pour the powder into a silver crucible containing about six grams of previously fused caustic potash. Reweigh the porcelain crucible to ascertain the amount of the residue actually taken for antimony, of course making correction for the difference later.

When fused, dissolve in water, add five grams tartaric acid and dilute hydrochloric acid to neutrality. Wash into flask and add five cc. strong sulphuric acid and one and a half grams potassium iodide, keeping the solution down to a bulk of IOo cc. as nearly as possible. Boil down rapidly to about fifty cc., observing the precautions already referred to, dilute with boiling water to about $100 \mathrm{cc}$, and boil down again to about fifty cc. The antimony is now all reduced.

Cool, add a pinch of dry phenol phthalein and caustic soda to alkalinity. Discharge the red color with dilute hydrochloric acid, cool again, add starch paste and fifty cc. saturated sodium bicarbonate and titrate with decinormal iodine solution to appearance of the blue color. We find that by working in this manner one cc. of the iodine is equivalent to 0.006 I 5 gram antimony. Theory requires 0.0060 . 
The tin is obtained by the difference in weight, calculating the antimony to $\mathrm{Sb}_{2} \mathrm{O}_{4}$. From every 20 per cent. tin so obtained, subtract one-tenth per cent., as explained previously.

In conclusion, we may add that any scheme which ignores the antimony passing into the nitric acid solution when the alloy is oxidized, is not worth considering. Working on one gram it amounts to from three to four per cent. of antimony, calculated on the alloy. Some results point to the feasibility of making correction for this solubility instead of holding back the main precipitate for it.

THE DUQUESNE CHEMTCAL LABORATORY, Pitssburg, Pa.

[CONTRIBUTION FROM THE JOHN HARRISON LABORATORY OF CHEMISTRY, No. 24.]

\section{THE CONSTITUTION OF ARSENOPYRITE.}

BY F. W. STARKE, H. I, SHOCK, AND EDGAR F, SMITH.

Received October 30,1897 .

THIS mineral is generally described as a sulpharsenide of iron. It has never been determined in what state of oxidation the iron exists, although it is customary to express the composition of the mineral by the formula FeSAs, in which some chemists have thought that the arsenic functions as a dyad element, that arsenopyrite consequently is nothing more than ordinary pyrite, in which an atom of arsenic has replaced an atom of sulphur, or that it is an iron arsenide in which sulphur, acting with a valence of three, has replaced arsenic. There is no experimental evidence favoring either assumption.

Pyrite and marcasite have been studied with the view of ascertaining the condition of the iron in them.' It was discovered that the first contained about one-fifth of its total iron content in the ferrous condition, whereas all the iron in marcasite existed in the ferrous state. Subsequent experiments have amply confirmed this observation. Efforts were also made to determine the nature of the iron in arsenopyrite. ${ }^{2}$ These resulted negatively.

The present communication presents experimental results

1 A. P. Brown: Am. Phil. Soc. Proc., 1894.

2 T. M. Lightfoot: This Journal, Sept., I 894. 